\title{
Performance of Fast-Neutron Imaging Detectors Based on Plastic Scintillator EJ-299-34
}

February 28, 2013

Prepared by

R. J. Newby, P. A. Hausladen, M. B. Blackston, J. F. Liang

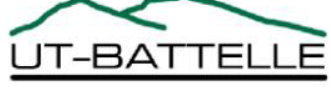




\section{DOCUMENT AVAILABILITY}

Reports produced after January 1, 1996, are generally available free via the U.S. Department of Energy (DOE) Information Bridge:

Web Site: http://www.osti.gov/bridge

Reports produced before January 1, 1996, may be purchased by members of the public from the following source:

National Technical Information Service

5285 Port Royal Road

Springfield, VA 22161

Telephone: 703-605-6000 (1-800-553-6847)

TDD: $703-487-4639$

Fax: 703-605-6900

E-mail:info@ntis.fedworld.gov

Web site: http://www.ntis.gov/support/ordernowabout.htm

Reports are available to DOE employees, DOE contractors, Energy Technology Data Exchange (ETDE), and International Nuclear Information System (INIS) representatives from the following sources:

Office of Scientific and Technical Information

P.O. Box 62

Oak Ridge, TN 37831

Telephone: 865-576-8401

Fax: 865-576-5728

E-mail: report@osti.gov

Web site:http://wwW.osti.gov/contact.html

This report was prepared as an account of work sponsored by an agency of the United States Government. Neither the United States nor any agency thereof, nor any of their employees, makes any warranty, express or implied, or assumes any legal liability or responsibility for the accuracy, completeness, or usefulness of any information, apparatus, product, or process disclosed, or represents that its use would not infringe privately owned rights. Reference herein to any specific commercial product, process, or service by trade name, trademark, manufacturer, or otherwise, does not necessarily constitute or imply its endorsement, recommendation, or favoring by the United States Government or any agency thereof. The views and opinions of authors expressed herein do not necessarily state or reflect those of the United States Government or any agency thereof. 
ORNL/TM-2013/82

Nuclear Security and Isotope Technology Division

\title{
Performance of Fast-Neutron Imaging Detectors Based on Plastic Scintillator EJ-299-34
}

\author{
R. J. Newby, P. A. Hausladen, M. B. Blackston, J. F. Liang
}

Date Published: February 28, 2013

\author{
Prepared by \\ OAK RIDGE NATIONAL LABORATORY \\ P.O. Box 2008 \\ Oak Ridge, Tennessee 37831-6285 \\ managed by \\ UT-Battelle, LLC \\ for the \\ U.S. DEPARTMENT OF ENERGY \\ under contract DE-AC05-00OR22725
}





\section{Contents}

LIST OF FIGURES $\ldots \ldots \ldots \ldots \ldots \ldots \ldots \ldots \ldots \ldots \ldots \ldots$

LIST OF TABLES $\ldots \ldots \ldots \ldots \ldots \ldots \ldots \ldots \ldots \ldots \ldots$. . . . . . . . . . . . . . . . . . .

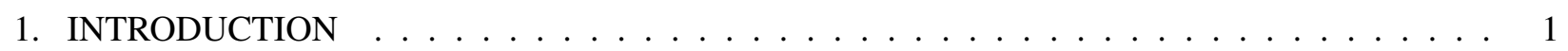

2. DETECTOR DESCRIPTION $\ldots \ldots \ldots \ldots \ldots \ldots \ldots \ldots \ldots \ldots$

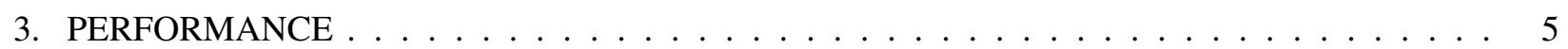

3.1 Pixel Separation . . . . . . . . . . . . . . . . . . . . . . 6

3.2 Pixel-by-Pixel Gain Corrections $\ldots \ldots \ldots \ldots \ldots \ldots \ldots$

3.3 Detector Uniformity $\ldots \ldots \ldots \ldots \ldots \ldots \ldots$

3.4 Neutron/Gamma Separation $\ldots \ldots \ldots \ldots \ldots \ldots$

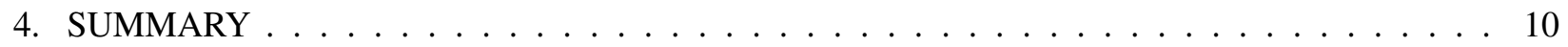

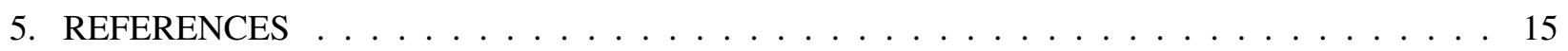





\section{List of Figures}

$1 \quad$ EJ-299-34 8×8 detector photographs $\ldots \ldots \ldots \ldots \ldots \ldots$

$2 \quad$ EJ-299-34 light emission curves for neutrons and gammas $\ldots \ldots \ldots \ldots$

$3 \quad$ Pixel-by-pixel pulse height spectrum of the ${ }^{137} \mathrm{Cs}$ Compton edge $\ldots \ldots \ldots \ldots$

$4 \quad$ Pulse height distribution vs neutron TOF $\ldots \ldots \ldots \ldots \ldots$

$5 \quad$ Flood image comparison $\ldots \ldots \ldots \ldots \ldots \ldots \ldots$

$6 \quad$ Nearest neighbor distance distribution comparison $\ldots \ldots \ldots \ldots \ldots \ldots$

$7 \quad$ Pixel-by-pixel calibrated ${ }^{137} \mathrm{Cs} \mathrm{spectrum} \ldots \ldots \ldots \ldots \ldots \ldots$

$8 \quad$ Block summed ${ }^{137} \mathrm{Cs}$ Compton edge comparison $\ldots \ldots \ldots \ldots \ldots$

$9 \quad$ Light collection uniformity $\ldots \ldots \ldots \ldots \ldots \ldots \ldots$

$10 \quad$ Pulse shape versus energy $\ldots \ldots \ldots \ldots \ldots \ldots \ldots \ldots \ldots$

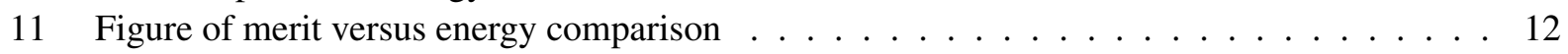

12 Neutron detection and identification efficiency $\ldots \ldots \ldots \ldots \ldots$ 



\section{List of Tables}

$1 \quad$ Pixel Separation Comparison $\ldots \ldots \ldots \ldots \ldots \ldots$ 



\section{INTRODUCTION}

The present report quantifies performance of the first fast-neutron imaging detectors constructed from the experimental plastic scintillator EJ-299-34 with neutron-gamma pulse-shape discrimination properties. The pixelated imaging detectors will form the position-sensitive detector plane of a coded aperture fast neutron imager, presently under construction at Oak Ridge National Laboratory (ORNL), intended for performing quantitative measurements of plutonium holdup at fuel-cycle facilities. Fast-neutron imaging is an appealing mode of measurement in scenarios where contact measurements with non-imaging detectors may be impractical or their results ambiguous because of surrounding material or nearby sources. The use of fast neutrons (as opposed to the more numerous gamma rays) is appealing because of their ability to penetrate equipment and nuclear material that is opaque to gamma radiation. In instances where neutron emanations are sufficiently numerous, neutron measurements may be better able to give quantitative mass estimates of holdup than gamma-ray measurements.

Previously, ORNL developed a prototype fast-neutron imager where the active volume of the neutron detectors consisted of commercially available liquid scintillator EJ-309. This imager was used to perform initial assessments of the utility of fast-neutron imaging for the purpose of quantifying plutonium holdup [1, 2, 3]. Experience with the liquid-scintillator-based imaging detectors indicates that they are a good laboratory device but are fragile to mechanical shock and temperature changes that are relatively modest (e.g., departure from room temperature by more than $20^{\circ} \mathrm{F}$ ). The fragility of the existing detectors to temperature changes, even for short durations or in storage, is highly undesirable. In addition, approval for use of liquid scintillators has historically been a problem in nuclear facilities, and as time passes, it is likely that this approval will get more (not less) difficult. In order for this technology to be usable in a production environment, replacement of the liquid-scintillator array with a plastic-scintillator array of comparable performance that is mechanically much more robust and does not contain flammable liquid is essential. Recently, plastic scintillator with pulse shape discrimination properties was developed by Lawrence Livermore National Laboratory and is now available from Eljen Technologies as experimental scintillator EJ-299-34 [4]. Pixelated imaging detectors have been built from the first production casting of this scintillator in plate form and evaluated for performance. This report summarizes the performance of the new detectors compared to the previous liquid scintillator detectors. The present report was prepared in fulfillment of milestone M4FT-13OR0402055, "Satisfactory detector performance" of Fuel Cycle R\&D Material Protection, Accounting, and Control Technology (MPACT) work package FT-13OR040205, "Fast-Neutron Imaging to Quantify Nuclear Materials - ORNL."

The remainder of the report is organized as follows. First, the physical construction of the detector is described. Then, characterization measurements using ${ }^{137} \mathrm{Cs}$ and ${ }^{252} \mathrm{Cf}$ are described and analyzed to determine the performance of the new plastic detectors. The measurements using the ${ }^{137} \mathrm{Cs}$ source were used to quantify the pixel separation, gain uniformity, and energy resolution of the detector. The

${ }^{252} \mathrm{Cf}$ measurement was used to measure the performance of the pulse shape measurement to distinguish neutrons from gamma rays and determine the detector efficiency. The new plastic-based detectors have better neutron-gamma discrimination, better average pixel separation, and more uniform light collection. 



\section{DETECTOR DESCRIPTION}

In this section, a brief description of the planned imager is given to provide context for the description of the neutron detector construction that follows.

The planned imager will consist of an aperture and a position-sensitive fast-neutron imaging detector whose relative positions are set with a linear stage in order to adjust the field of view. A high-density polyethylene (HDPE) aperture mask will be used to spatially modulate neutrons incident on the detector, and the pattern of modulation will be used to reconstruct an image of the incident radiation. For the HDPE aperture mask, a base-11 modified uniformly redundant array (MURA) pattern will be used [5]. The neutron detector portion of the imager will consist of a panel of nine individual detectors that pack closely together to give a total area of $33.7 \times 33.7 \mathrm{~cm}^{2}$ and comprise 576 individual pixels in a 24 pixel by 24 pixel array. The $24 \times 24$ array of detector pixels is sufficient to double-sample the apertures in the mask and is significantly fewer pixels than the 40 pixel by 40 pixel liquid-based prototype. This smaller number is intended to reduce cost, to achieve a more compact size for the imager, and to favor sensitivity over angular resolution.

The active volume of a single detector module, typically referred to as the "pixel block," consists of a $108 \times 108 \times 5 \mathrm{~mm}^{3}$ volume of scintillator EJ-299-34 segmented by 3 M Vikuiti reflector into an $8 \times 8$ array of $13.5 \times 13.5 \times 50 \mathrm{~mm}^{3}$ optically isolated pixels of plastic scintillator. The pixels are viewed through a 28-mm-thick segmented acrylic light guide by four $52 \mathrm{~mm}$ Hamamatsu R7724-100 super bialkali photomultiplier tubes (PMTs) whose shared response determines the pixel of interaction.

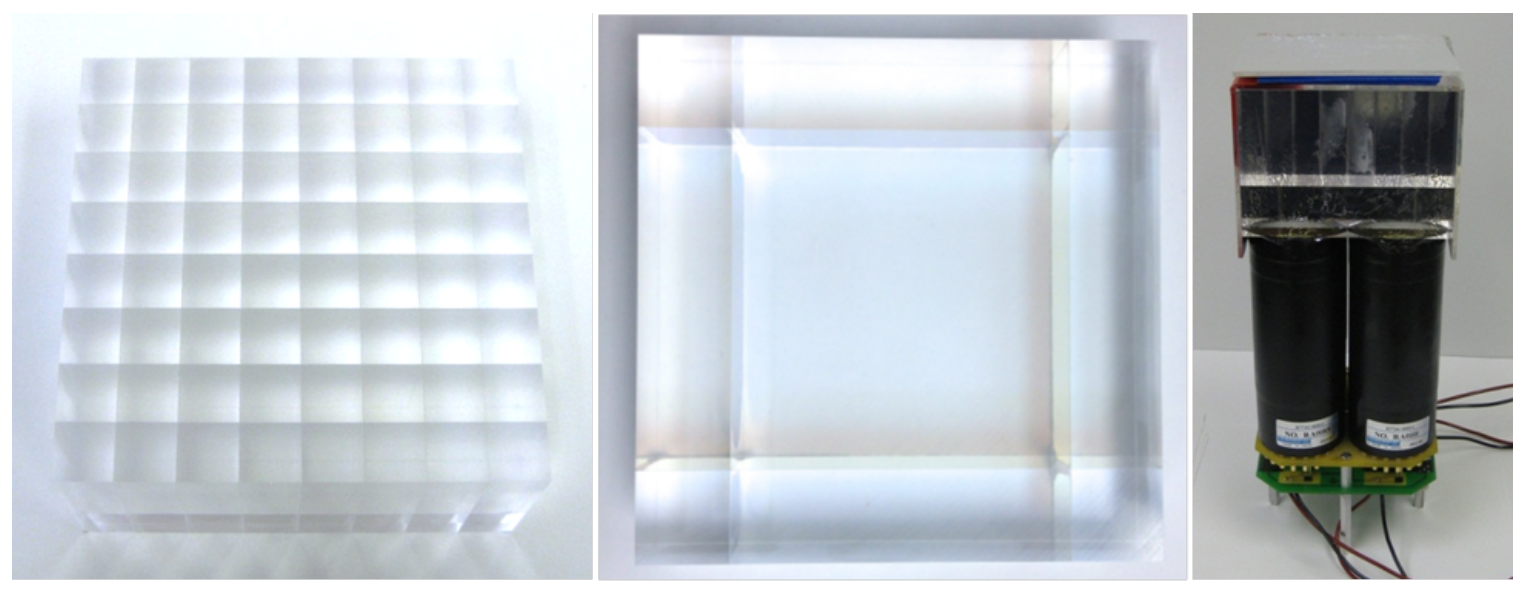

Figure 1. EJ-299-34 8 $\times 8$ segmented pixel array (left), PMMA light guide (middle), and assembled detector module in its test configuration (right).

The large length compared to transverse dimension of the pixels enables them to also function as a light pipe, where scintillation light acts on average as if it is emitted from the center of the open end facing the PMTs. The position of the scintillation event within the pixel array is calculated by Anger logic from the amount of light deposited in each PMT. The amount of light sharing among the PMTs is controlled by the light guide, both by its overall length and the optical segmentation within it. For the present work, the design of the light guide was optimized to maximize pixel separation and linearity of response across the detector consistent with a minimum number of segments in the light guide to minimize both cost and complexity. The detector module is placed in a housing whose external dimensions in the imaging plane are $112 \mathrm{~mm} \times 112 \mathrm{~mm}$. The active area therefore comprises $92 \%$ of the total, an increase of $10 \%$ over the 
previous liquid scintillator detectors. A photograph of the segmented EJ-299-34 scintillator pixel block, light guide, and assembled detector module are shown in Figure 1 .

In most neutron imaging applications, the total count rate in the detectors is dominated by the gamma-ray rate. All organic scintillators are sensitive to neutrons and gamma rays, so neutron-gamma discrimination is essential for performing quantitative measurements of neutron source strengths. To count neutron sources only, identification of neutron-induced pulses is achieved via pulse-shape discrimination (PSD). To maximize PSD performance, a PMT was chosen that enables a more faithful measurement of pulse shape. Hamamatsu R7724-100 PMTs enable more faithful measurement of pulse shape because the super bialkali photocathode enhances photoelectron statistics and the low afterpulsing design of the PMT eliminates most of the anomalous current that can follow a pulse in a conventional PMT, confounding the measurement of pulse shape. For these characterization measurements, 200 samples ( $800 \mathrm{ns)}$ of each pulse were recorded for each PMT using four channels of a Struck SIS3316 14-bit $250 \mathrm{MSs}^{-1}$ waveform digitizer. The individual PMT signals are evaluated to determine the position of interaction in the detector, while the pulse shape is extracted from the sum of the four signals. A plot of the average pulse shapes for neutrons (blue) and gamma rays (red) is shown in Figure 2. From these data, detector performance will be assessed and compared to the previously characterized liquid scintillator detectors.

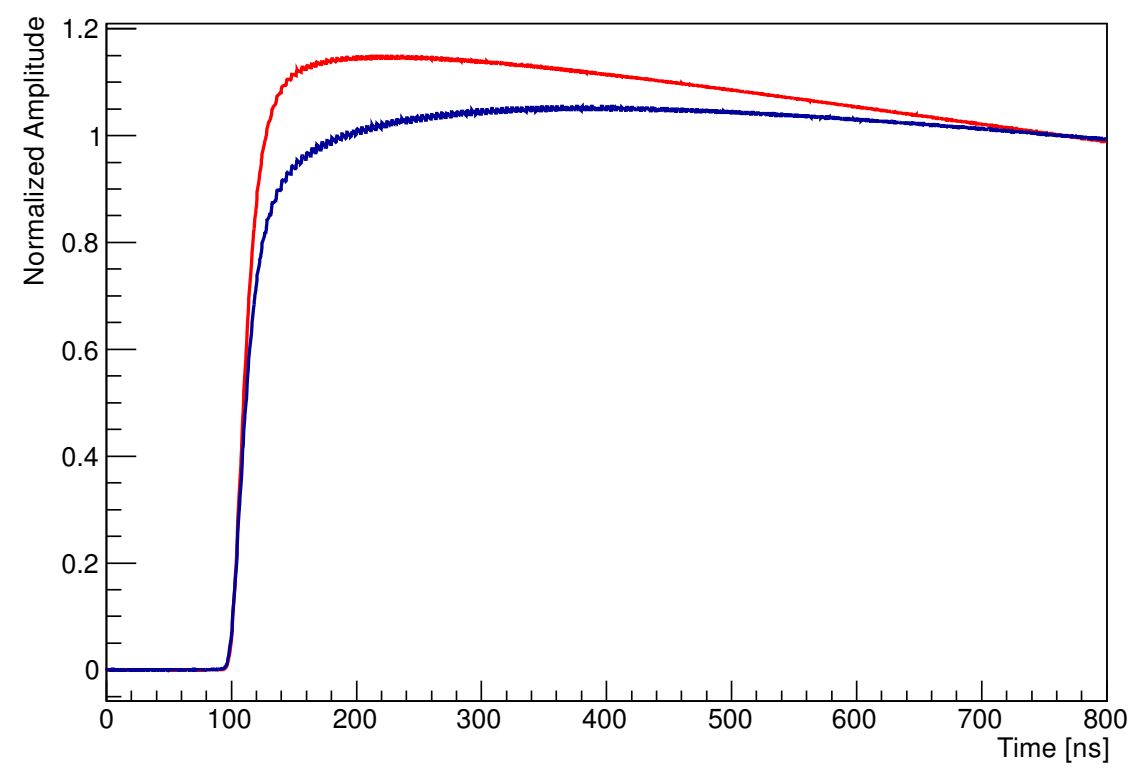

Figure 2. Average light curves for neutrons (blue) and gammas (red) as recorded with a Struck 3316 waveform digitizer and an integrating preamplifier $(\tau=2.4 \mu \mathrm{s})$ normalized to the amplitude $660 \mathrm{~ns}$ after the initial rise. 


\section{PERFORMANCE}

The essential performance metrics of the pixelated detector block for this application are position resolution and neutron identification. Detector uniformity is also important since any detector variations will require systematic corrections. Any uncertainties in large corrections may introduce undesired imaging features. Adequate energy resolution is also necessary to optimize neutron identification. These metrics are evaluated in the analysis of the ${ }^{137} \mathrm{Cs}$ and ${ }^{252} \mathrm{Cf}$ measurements.

For the ${ }^{137} \mathrm{Cs}$ measurement, the detector block is exposed to a ${ }^{137} \mathrm{Cs}$ source to record the energy depositions in the Compton scattering of $662 \mathrm{keV}$ gamma rays. After gain matching the PMTs, a 4 hour data set was recorded using a ${ }^{137} \mathrm{Cs}$ source with an activity of $1 \mu \mathrm{Ci}$ positioned so as to record approximately 175 counts per second in the detector module to provide approximately 38,000 counts in the pulse-height spectrum associated with each pixel. These data are used to calibrate the position reconstruction, pixel-by-pixel gain matching, and pixel-by-pixel energy calibration and are discussed below in the corresponding sections.

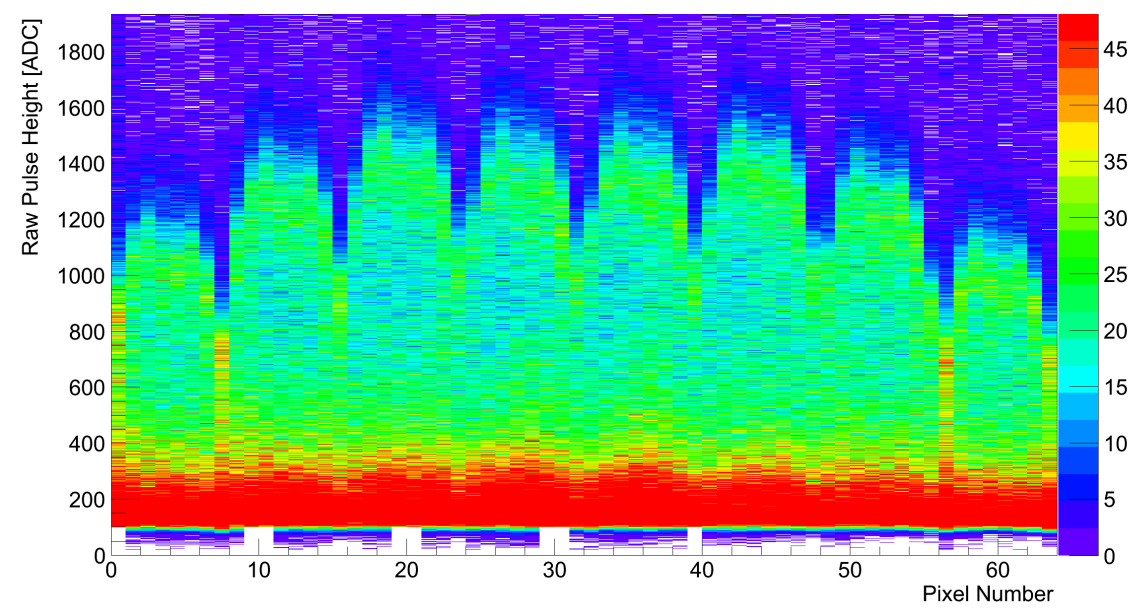

Figure 3. Pixel-by-pixel pulse height spectrum of the ${ }^{137}$ Cs Compton edge.

For the ${ }^{252} \mathrm{Cf}$ measurement, a neutron time-of-flight measurement was performed using a time-tagged ${ }^{252} \mathrm{Cf}$ fission neutron and gamma-ray source having a fission activity of 4800 fissions per second (as of February 2013). The TOF measurement provided a means for calibrating the pulse-shape discrimination cuts, determining the energy dependence of the absolute neutron efficiency, and quantifying the level of gamma contamination for identified neutrons as a function of relative neutron efficiency.

In the time-tagged source, the ${ }^{252} \mathrm{Cf}$ is located on one plate of a parallel-plate ion chamber, allowing a timing signal to be generated by the ionization induced by energy loss from one or both of the fission fragments slowing in the ion chamber fill gas [6]. This induced signal was amplified and a logic signal generated by means of a constant-fraction discriminator. The four PMTs that read out the detector panel are recorded by four channels of the Struck 3316 waveform digitizers having modified firmware to implement a digital constant fraction discriminator on the sum of the individually digitized signals. To perform the TOF measurement, a fifth channel of the digitizer recorded timing signals from the ion chamber with a firmware setting to use the same digital constant fraction algorithm on the single signal. The neutron source was placed approximately $89 \mathrm{~cm}$ from the detector center, and the neutron detector and 
ion chamber signals were recorded for a period of 12 hours. A two-dimensional histogram of the number of counts as a function of TOF and pulse height is shown in the top panel of Figure 4, with the corresponding time-of-flight calculated neutron energy in the bottom panel. We estimate from the gamma timing distribution a resolution of 3 ns FWHM.
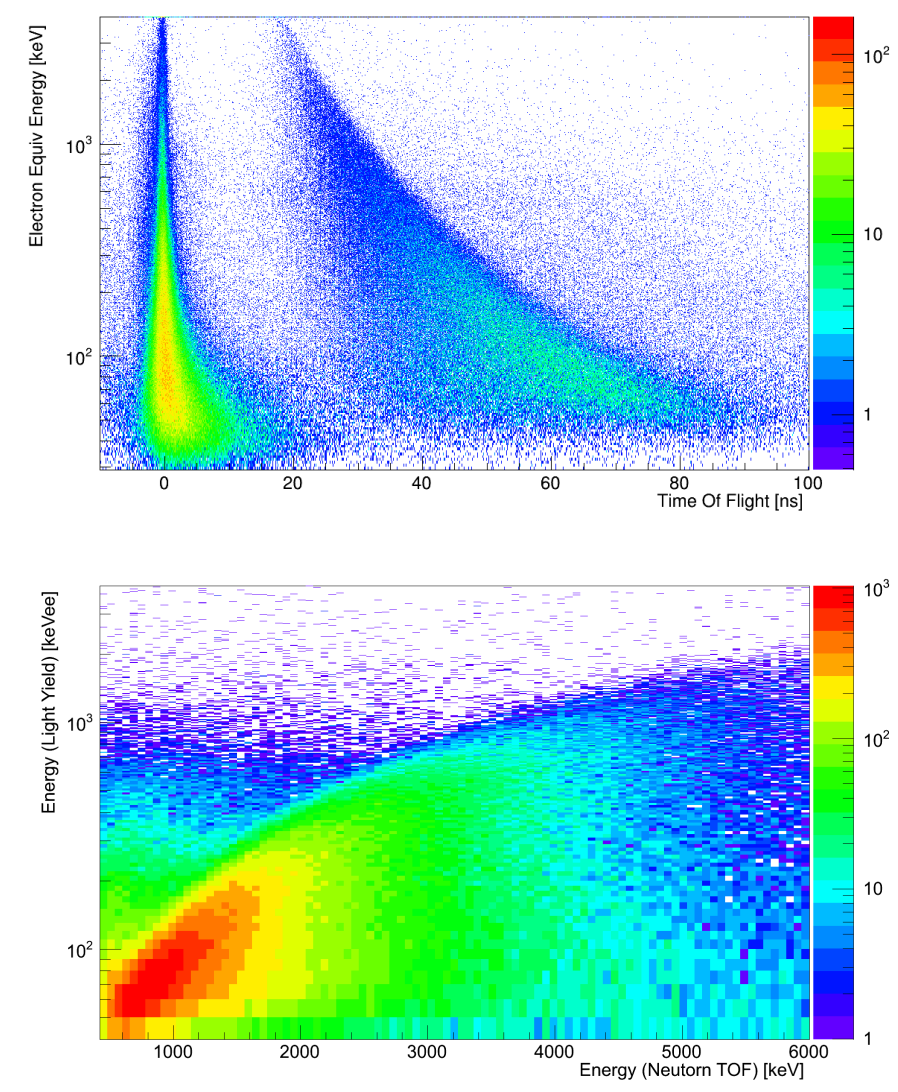

Figure 4. The pulse height distribution vs time of flight (top) and calculated neutron energy (bottom).

\subsection{Pixel Separation}

The position reconstruction was calibrated using the ${ }^{137} \mathrm{Cs}$ data set. The light sharing distribution of the four PMTs was projected onto a two-dimensional plane as shown in the left panel of Figure 5. Peaks in the distribution corresponding to all 64 pixels are clearly visible. An automated algorithm was used to find each peak, fit the centroid, and divide the plane into regions associated with each pixel of the array. These defined regions are then used to identify individual events with a single pixel. The right panel of Figure 5 is the light sharing distribution from a typical $10 \times 10$ liquid scintillator detector for comparison.

Good detector designs maximize the distance between the peaks corresponding to individual pixels in the measured position response. To quantify how well a particular design performs, we can measure the distribution of the distance to nearest-neighbor pixels. Better designs correspond to larger mean and smaller root-mean-squared (RMS) distances. A quantitative comparison of the $8 \times 8$ EJ-299-34 detector and the $10 \times 10$ EJ-309 detector is made in Figure 6 . The uniformity of the $8 \times 8$ detector is much improved over 

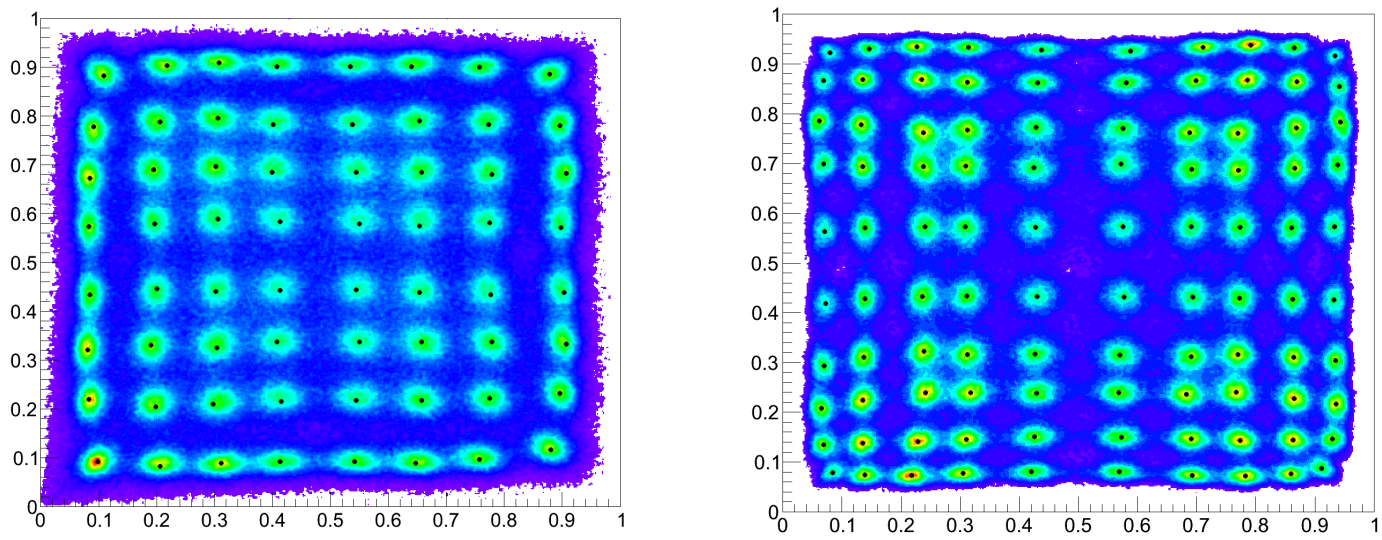

Figure 5. Flood images of the $8 \times 8 \mathrm{EJ}-299-34$ plastic block detector (left) and the $10 \times 10 \mathrm{EJ}-309$ liquid block detector (right).

the $10 \times 10$ detector; the amount of improvement is more than expected from having fewer pixels alone. The reason for this is that the four pixels in the center of the $10 \times 10$ detector have much larger separation, driving the rest of the pixels into closer proximity. This wide spacing is absent in the $8 \times 8$ detector. The average separation and fractional variation are shown in Figure 6 , tabulated in Table 1 , and indicate an improvement of a factor of 2.5 in the uniformity and $13 \%$ in the average distance.

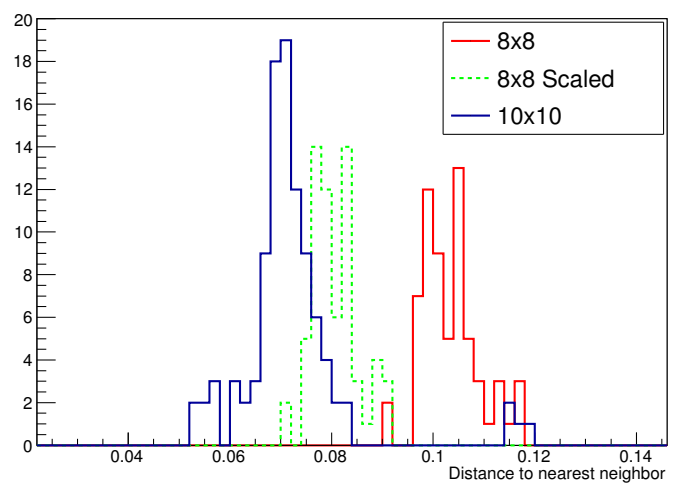

Figure 6. Pixel position nearest-neighbor distributions for the $8 \times 8 \mathrm{EJ}-299-34$ plastic block detector (red) and the $10 \times 10 \mathrm{EJ}-309$ liquid block detector (blue). The $8 \times 8$ distribution is scaled (green) by the ratio of the number of inter-pixel spaces for better comparison with the $10 \times 10$ block.

\subsection{Pixel-by-Pixel Gain Corrections}

Gain matching of the individual pixels and energy calibration were performed using the detector response to Compton scatters of the $662 \mathrm{keV}$ gamma ray emitted from a ${ }^{137} \mathrm{Cs}$ source. The maximum energy that can be imparted to an electron in a single scatter, or "Compton edge," of each of the detector pixels was identified and aligned to the value of $477 \mathrm{keV}$ expected for $662 \mathrm{keV}$ gamma rays. Because of 
Table 1. Distance to nearest neighboring pixel

\begin{tabular}{|l|c|c|c|}
\hline & $8 \times 8$ & $8 \times 8$ Scaled & $10 \times 10$ \\
\hline Mean & 0.104 & 0.081 & 0.072 \\
\hline RMS (\%) & 6 & 6 & 15 \\
\hline
\end{tabular}

the large number of pixels shown in Figure 7, the Compton edge was identified via an automated algorithm intended to find the steepest slope on the pulse-height spectrum in the region of the edge; computationally, this was performed by computing the negative derivative of the spectrum and finding its maximum.

Because of statistical fluctuations of the counts in any one energy bin, it was necessary to smooth this derivative by 40 channels.

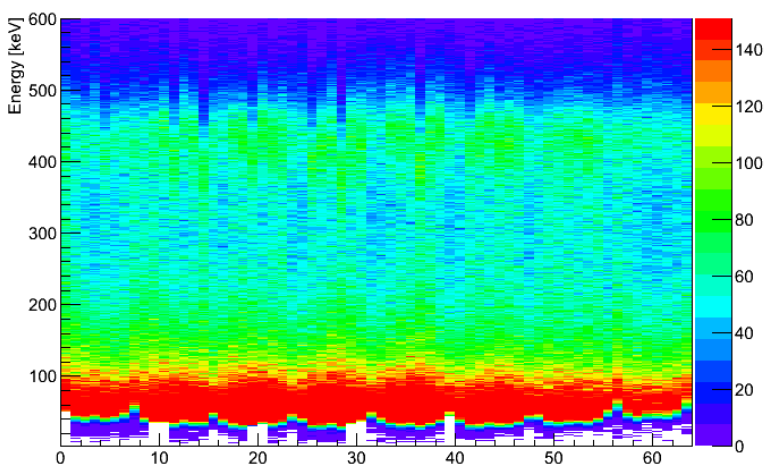

Figure 7. Calibrated ${ }^{137} \mathrm{Cs}$ pulse-height spectrum (y-axis) for each of the 64 pixels (x-axis).

With the Compton edges aligned to $477 \mathrm{keV}$, the pulse height spectra were calibrated for the response of electrons in "keV electron-energy equivalent" or keVee. The calibrated pulse height spectrum is shown in blue in the left panel of Figure 8. The $477 \mathrm{keV}$ Compton edge is clearly visible in the spectrum, and by considering the slope of the edge in the calibrated spectrum, the resolution of the detector is estimated to be no worse than $15 \%$ and is slightly improved compared to the resolution observed in the EJ-309 liquid scintillator block detectors overlaid in red in the right panel of Figure 8.

\subsection{Detector Uniformity}

In the process of gain-matching the individual pixels, the relative efficiency for collecting scintillation light is known on a pixel-by-pixel basis. The efficiency of collecting scintillation light is not uniform across the detector, with systematically lower efficiency of light collection for edge and corner pixels. This position-dependent efficiency for light collection results from a variety of factors, but one of the most significant relates to properties of the $3 \mathrm{M}$ Vikuiti reflector used in the pixel block and light guide. While this reflector has excellent reflectivity for light greater than $400 \mathrm{~nm}$ wavelength, it transmits rather than reflects scintillation light shorter in wavelength than $400 \mathrm{~nm}$. For the central detector pixels, light transmits through the reflector and into neighboring pixels where it can still be detected. For edge and corner pixels, light that transmits through the reflector and out of the detector is lost. 

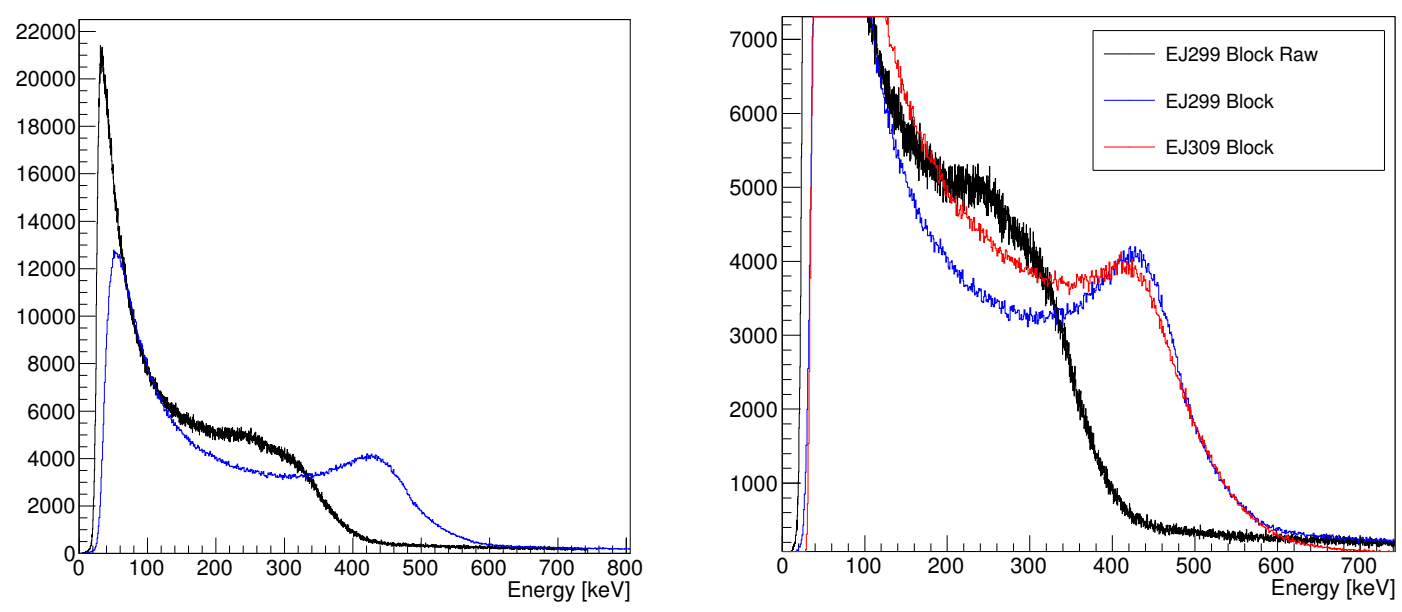

Figure 8. The ${ }^{137} \mathrm{Cs}$ Compton spectra after before (black) and after (blue) matching. Overlaid is a similar spectrum from the EJ-309 $10 \times 10$ liquid scintillator block detector.

The plastic block detector is expected to have less variation in light collection than the previous liquid detectors. Indeed, the observed ratio of the center pixel gains to the corner pixel gains has been reduced from a factor of 3 in the liquid detector to a factor of 2 in the plastic detector as demonstrated in Figure 9. In its final configuration (rather than test configuration reported here), the amount of gain variation originating from poor light collection from edge and corner pixels should be further reduced. This reduction in gain variation will be accomplished first, by wrapping the pixel block and light guide with Teflon so that light penetrating the Vikuiti reflector need not be lost, then by covering with reflector the back corners of the light guide that are not covered by the PMTs .
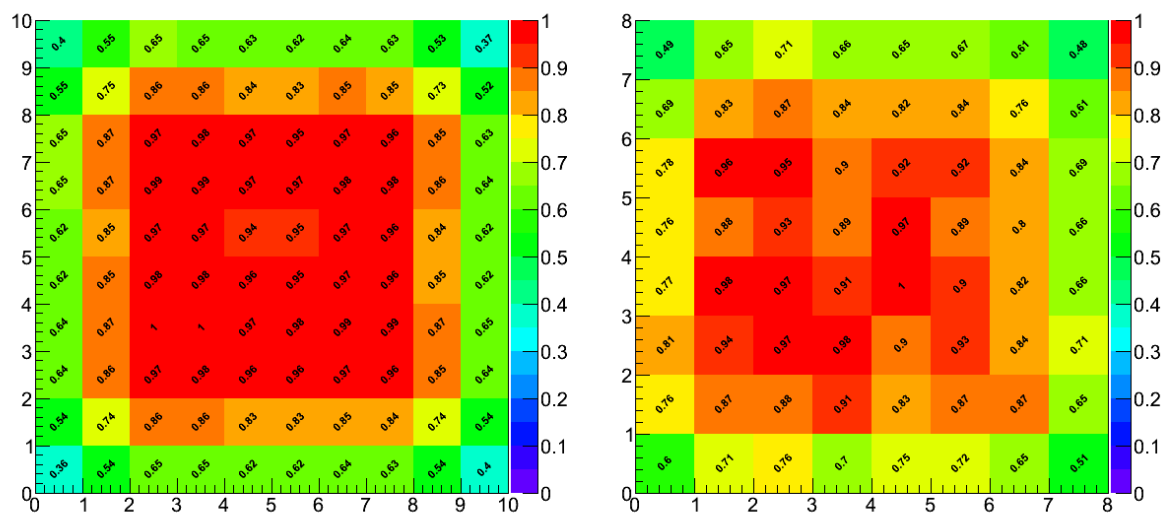

Figure 9. Gain variations are extracted from a pixel-by-pixel measure of the ${ }^{137} \mathrm{Cs}$ Compton edge. 


\subsection{Neutron/Gamma Separation}

The discrimination of neutrons from gammas relies upon the distinct time dependence of the scintillation light for the corresponding recoil proton and Compton electron. Differences in pulse shape for neutron and gammas are evident in the average waveforms as recorded with the plastic block detector with an integrating preamplifier of $\tau=2.4 \mu$ s shown in Figure 2 normalized to the amplitude $660 \mathrm{~ns}$ after the initial rise. A pulse shape parameter is calculated from the digitized waveform of each interaction by taking the ratio of the prompt light to the total light. The prompt light fraction $P_{\gamma}$ measured for gamma interactions forms (to first approximation) a binomial distribution with a mean, $\mu_{\gamma}=N \cdot P_{\gamma}$, determined by the relative contribution of the prompt light to total characteristic of Compton electrons and a width,

$\sigma_{\gamma}=\sqrt{N \cdot P_{\gamma}\left(1-P_{\gamma}\right)}$, where $N$ is the total number of photons. The prompt light fraction measured for neutrons has a mean, $\mu_{n}$, and width, $\sigma_{n}$, characteristically different from the gammas. We optimize the time to sample the prompt light to maximize the figure of merit (FOM) on a subset of the collected data with electron equivalent energies $350-450 \mathrm{keV}$ where the figure of merit is defined as follows:

$$
\mathrm{FOM} \equiv \frac{\mu_{\gamma}-\mu_{n}}{2.35\left(\sigma_{\gamma}+\sigma_{n}\right)}
$$

As can be inferred from Figure 2 , the figure of merit is maximized by sampling the prompt light at 48 ns after the beginning of the pulse. The energy dependence of the optimized pulse-shape parameter is measured for neutrons and gammas shown in Figure 10 . The pulse shape distributions are divided into slices in energy such that the number of neutrons for each energy bin is about the same. Each energy bin is fit with a double Gaussian function to estimate the means and widths of the neutron and gamma bands. The typical neutron selection criteria includes a band within $2 \sigma_{n}$ from the neutron mean and excludes any event within of $5 \sigma_{\gamma}$ of the gamma mean. The corresponding energy dependence of the figure of merit is compared with previous characterizations of the liquid scintillator block detectors in Figure 11. The new detectors perform as well as the typical liquid detector and better at electron equivalent energies below 500 keVee at which the bulk of fission neutrons are emitted.

Neutrons and gammas are well separated by time of flight which provides an independent means of particle identification with which to evaluate the pulse-shape discrimination for gamma rejection and neutron efficiency. We find that for times corresponding to gammas (0-10 ns), the pulse-shape discrimination effectively rejects $99.9 \%$ of ${ }^{252} \mathrm{Cf}$ gammas interacting in the detector while at the same time maintaining a neutron efficiency of $17.5 \%$ averaged over the ${ }^{252} \mathrm{Cf}$ spectrum.

Using the measured time of flight, we calculated the neutron kinetic energy to determine the energy dependence of the absolute neutron efficiency within the solid angle of the detector. In Figure 12, we evaluate both the neutron detection efficiency (red) and the additional losses resulting from imposing sufficient gamma rejection (green). The detection efficiency remains flat down to about $1 \mathrm{MeV}$ at which the light amplitudes fall below the hardware triggering thresholds. The gamma rejection, however, cuts steepest at about 1.2 MeV. This neutron energy corresponds to about $100 \mathrm{keVee}$ where the gamma rejection cut crosses the neutron band.

\section{SUMMARY}

We have completed the construction of the first pixelated imaging detectors using the experimental EJ-299-34 and evaluated the performance metrics relevant for the final imager including position 


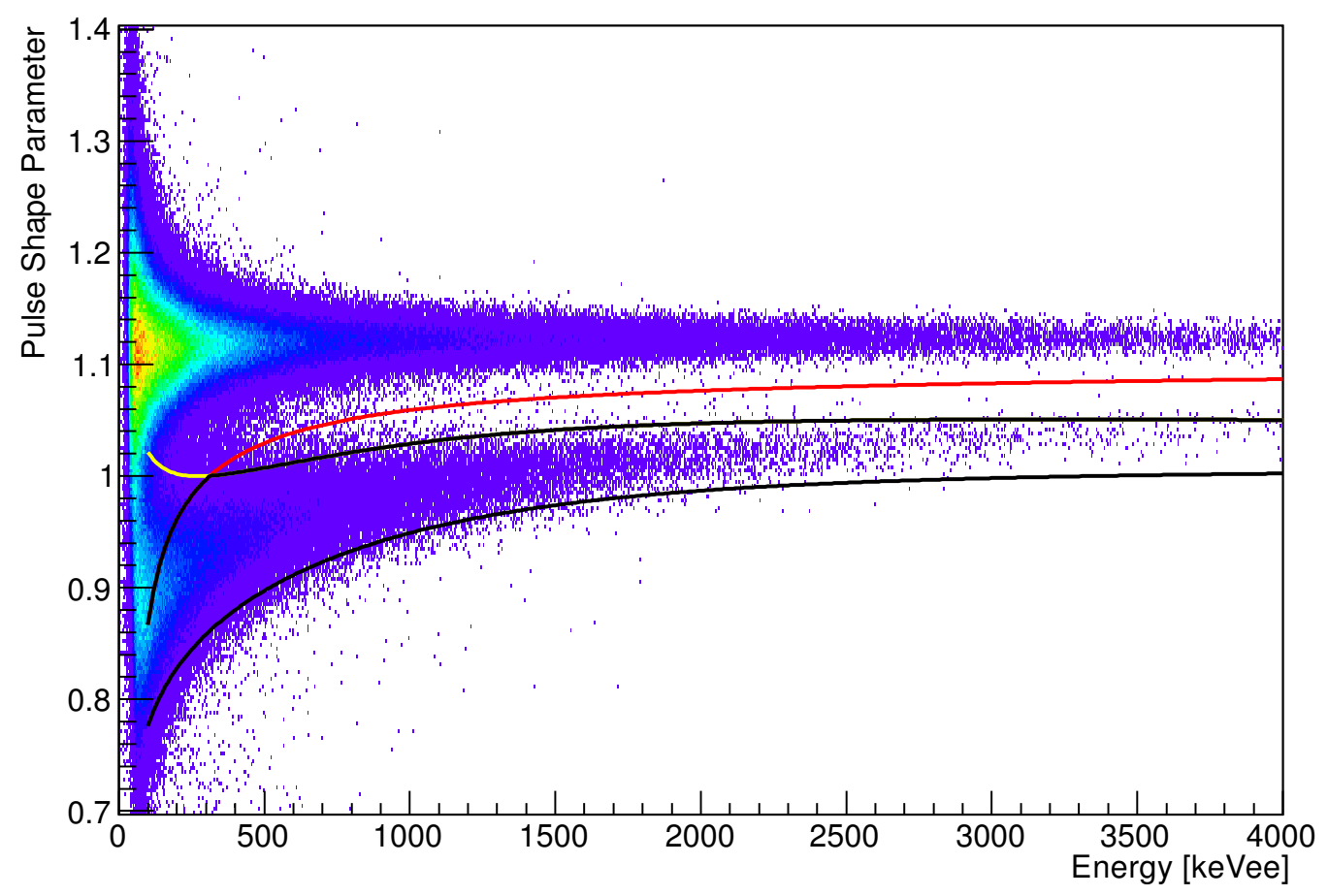

Figure 10. Pulse shapes characterized by the ratio of the the prompt integral light to the total integral light. Pulse shape versus energy is overlaid with the $2 \sigma$ neutron inclusive cut (black) and the $5 \sigma$ gamma exclusion cut (red).

resolution, neutron identification, detector uniformity, and energy resolution. We find the detector to perform satisfactorily in each of these metrics. 


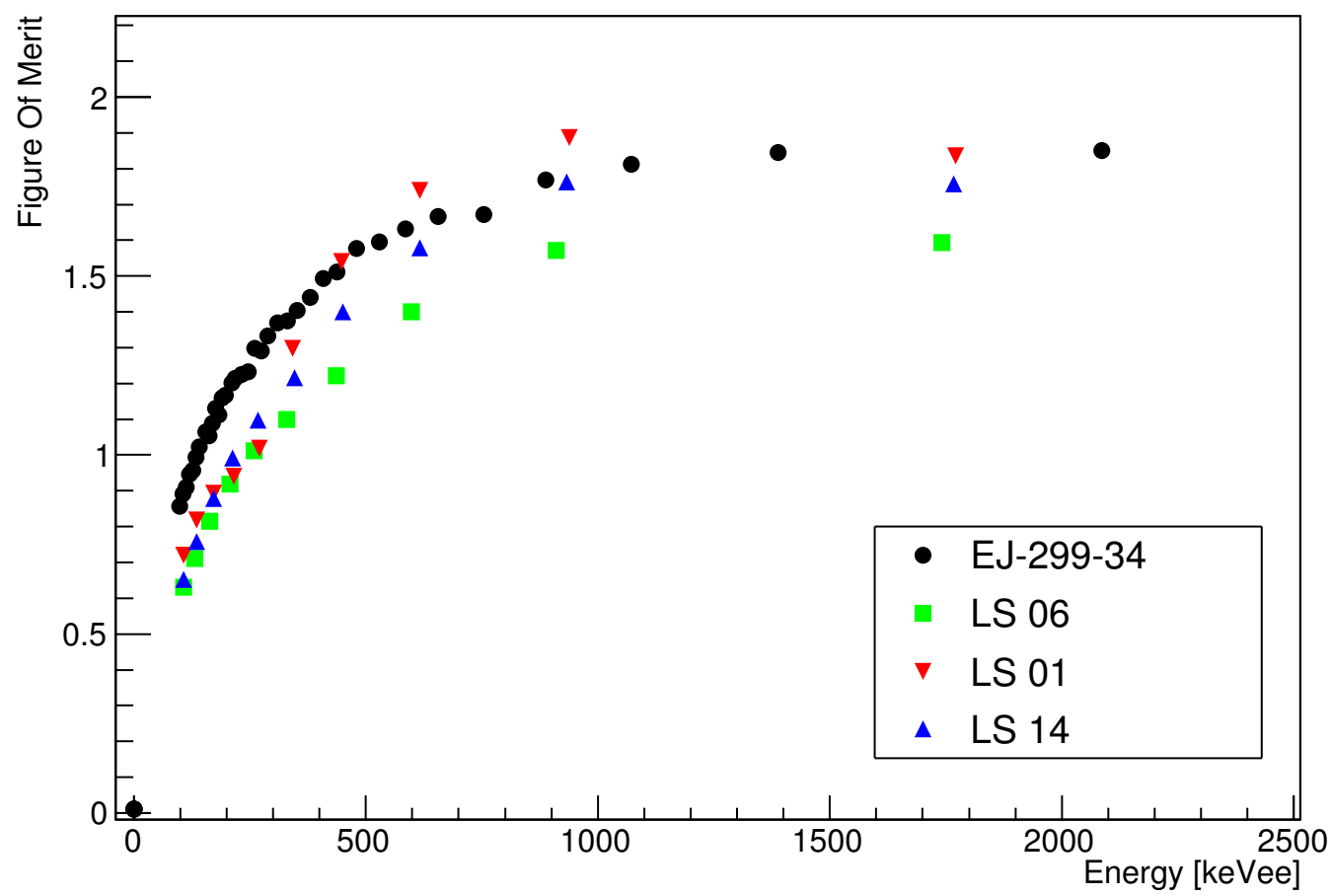

Figure 11. Figure of merit versus energy for the EJ-299-34 block detector and three representative EJ-309 liquid scintillator block detectors. 


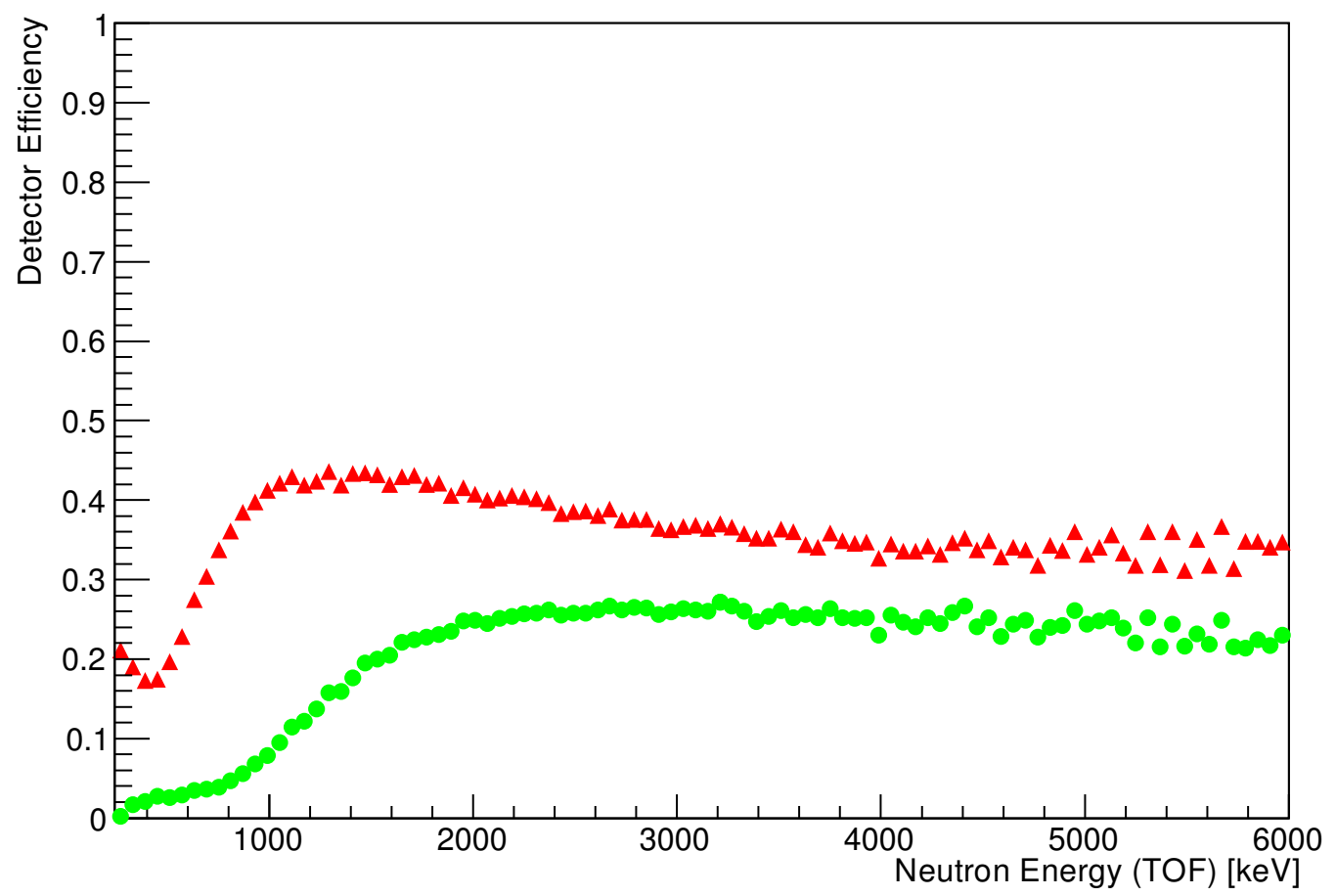

Figure 12. Neutron detection (red triangles) and identification (green circles) efficiencies as a function of neutron energy as determined by time of flight. 



\section{REFERENCES}

[1] P. A. Hausladen, M. A. Blackston, and R. J. Newby, Position-Sensitive Fast-Neutron Detector Development in Support of Fuel-Cycle R\&D MPACT Campaign, ORNL/TM 2010/201.

[2] P. A. Hausladen, M. A. Blackston, and R. J. Newby, Demonstration of Emitted-Neutron Computed Tomography to Quantify Nuclear Materials, ORNL/TM 2011/357.

[3] Paul Hausladen, Matthew Blackston, Felix Liang, and Jason Newby, Measurements and Analysis of Mock Holdup Configurations Using Fast-Neutron Imaging, ORNL/TM 2012/400.

[4] Natalia Zaitseva, Benjamin L. Rupert, Iwona Pawełczak, Andrew Glenn, H. Paul Martinez, Leslie Carman, Michelle Faust, Nerine Cherepy, Stephen Payne, "Plastic scintillators with efficient neutron/gamma pulse shape discrimination," Nuclear Instruments and Methods in Physics Research A 668, 88 (2012).

[5] S. R. Gottesman and E. E. Fenimore, "New family of binary arrays for coded aperture imaging," Applied Optics, Vol. 28, No. 20, 4344-4352 (1989).

[6] L. G. Chiang, A. C. Gehl, J. K. Mattingly, J. A. McEvers, J. T. Mihalczo, J. A. Mullens, R. B. Oberer, Nuclear Materials Identification System Operations Manual, ORNL/TM-2001/65, Rev. 2, pp. 5-7. 